\title{
Rank Average for handover decision making in heterogeneous wireless networks
}

\author{
Saida Driouache ${ }^{1, *}$, Najib Naja $^{1}$, Abdellah Jamali ${ }^{12}$ \\ ${ }^{1}$ STRS laboratory, INPT, Rabat, Morocco \\ ${ }^{2}$ IR2M laboratory, FST, Hassan 1st University, Settat, Morocco
}

\begin{abstract}
Vertical handover mechanism is an important issue in heterogeneous wireless networks. Continuity of service during handover and QoS are relevant issues to deal with. This paper focuses on vertical handover decision making. It proposes a Rank Average method for the best access network selection. This method is an aggregation of two Multi Attributes Decision Making (MADM) methods: Technique for Order Preference by Similarity to Ideal Solution (TOPSIS) and VIse Kriterijumska Optimizacija kompromisno Resenja (VIKOR), together with Shannon entropy-based weights. Entropy is an adequate tool to weigh up the handover criteria in a quiet easy and straightforward way. Rank Average outperforms TOPSIS and VIKOR in terms of throughput, end to end delay, packet loss, and significantly reduces the number of unnecessary handovers related to the ping-pong effects. Performance results are obtained within the scope of MIH through NS3 simulator.
\end{abstract}

Received on 31 May 2017; accepted on 12 November 2017; published on 10 January 2018

Keywords: heterogeneous networks, vertical handover, quality of service, continuity of service

Copyright (C) 2018 Saida Driouache et al., licensed to EAI. This is an open access article distributed under the terms of the Creative Commons Attribution license (http://creativecommons.org/licenses/by/3.0/), which permits unlimited use, distribution and reproduction in any medium so long as the original work is properly cited.

doi:10.4108/eai.10-1-2018.153555

\section{Introduction}

The evolution of heterogeneous access technologies, and real time multimedia applications has created a plenty of new wireless connectivity scenarios showing an increasing number of devices. So, the integration of Heterogeneous Networks (HetNets) in such a scenario, is offering better communication channels and raising the possibility of affording better Quality of Service (QoS). Vertical Handover (VH) occurs when a user moves among different networks. This process is divided into three steps: The first step is the network discovery when the terminal equipment identifies all the possible accessible networks. The second step is the decision of handover, when the terminal equipment selects the target network. The third step is the handover execution, when terminal equipment switches to the selected network. Seamlessness in network switching is still considered as one important challenge faced in the handover management process [1]. In order to be always best connected, the handover process should start at the appropriate time and select

*Corresponding author. Email: driouache@inpt.ac.ma the most adequate target interface. The design of such an intelligent $\mathrm{VH}$ algorithm is a challenge.

As a means of providing interoperability and seamless handover in HetNets, IEEE introduced a standard called IEEE 802.21 or MIH [2]. It allows IEEE and nonIEEE technologies to be integrated. At the same time, it ensures both vertical and horizontal handovers. It defines an entity called MIH Function (MIHF) as a generic interface between the different technologies of the link layer and the upper layers. Lower layers coordinate the exchange of information and commands between devices involved in the handover decision making. Each node has a set of MIHF users which are typically the protocols of mobility management. They use MIHF to monitor and collect information related to the handover.

Designating a network that satisfies user demands is another challenge, as some criteria may conflict with each other. The selection process becomes a multicriteria decision making problem [3]. The significance of this paper is that it proposes an aggregation approach, which mixes two MADM methods: TOPSIS and VIKOR. It uses the ranking results obtained from TOPSIS [4][5] and VIKOR [6][7]. It provides a rank 
average for each alternative (access network). we also employ the entropy method to obtain the objective weights of the criteria. Real time applications such as video streaming and VOIP demand rigorous QoS requirements. So, handover delay, throughput, end to end delay, and packet loss rate are used to measure QoS and network performance.

The remaining part of the paper is organized as follows: section 2 recaps related work. Section 3 presents implied MADM methods. The proposed selection method is presented in section 4 . Section 5 presents the calculated results, explains the simulation scenario, and compares the evaluation results of the Rank Average, TOPSIS, and VIKOR. Conclusion is provided in section 6.

\section{Related work}

Various VH algorithms [8] have been proposed. The usual policy of $\mathrm{VH}$ decision is based on Radio Signal Strength (RSS). Several RSS based algorithms have been suggested [9]. These simple implementations provide low handover latency but a low to medium throughput. The basic idea of a cost function based algorithm [10][22] is to define a cost based on a combination of network parameters. Consequently, the candidate systems performance can be weighed up, in order to select the best network. Generally, cost function algorithms do not yield a higher throughput than simple approaches. Besides, delays are due to the complexity during information collection and cost function calculation. Fuzzy logic and artificial neural networks [11][12], are widely used in the literature to make handover decisions. The application of these complicated algorithms is necessitated by the complexity of handover decisions and wireless networks dynamic conditions. The Context-Aware [9] handovers are based on informations related to the Mobile Terminal (MT), network, and other contextual factors. These informations can include the network capacity, location, subscriber preferences, type of service, etc. MADM methods are extensively applied for real world problems [13].They have been adopted [2] in order to select the suitable access network for a handover. MADM techniques commonly used are: Simple Additive Weighting (SAW), TOPSIS [2], Analytic Hierarchy Process (AHP), GRA (Grey Relational Analysis), Weighted Sum Model (WSM), Weighted Product Method (WPM) or Multiplicative Exponent Weighting (MEW), ELimination Et Choix Traduisant la REalite (ELECTRE), VIKOR, and Linear Assignment. They combine informations in a problem decision matrix to ascertain a final ranking or selection from among the alternatives. Some MADM methods have been proposed [4][5] to make VH decisions. In [4], the author compares two MADM methods: TOPSIS and
SAW, in terms of handover delay. For many considered criteria, TOPSIS performance is good enough compared to SAW. Two classes of algorithms are considered [5]: Single Performance Metric Optimization (SPMO) and Multiple Performance Metric Optimization (MPMO). The MPMO techniques consider different performance attributes simultaneously during the selection process, and try to find a compromise between the used attributes. Among the MPMO approaches, TOPSIS algorithm shows satisfactory performance for all considered metrics. MADM algorithms provide a higher throughput. Unfortunately, the attributes evaluation complexity increases the handover delay. This is also true for more complex approaches such as AI and context-aware methods.

History of VH decision making exhibits helpful utilization of MADM tools [10]. To facilitate and assure a reliable access network selection, the experts evaluate and select target network by several metrics rather than single criterion. TOPSIS and VIKOR, are capable of attending in wide range of access network selection problems. Comparative study of VIKOR and TOPSIS has been performed in [20][23]. VIKOR, TOPSIS, PROMETHEE (Preference Ranking Organization METHod for Enrichment of Evaluations) and AHP [14] are applied to seek the most suitable target network [7][14]. Authors in [15] found out that the final ranking of alternatives vary across methods, especially in problems with many alternatives. TOPSIS and VIKOR use distinct normalization techniques [7] and introduce some aggregating functions for ranking. Authors in [16] compared the extended VIKOR method with TOPSIS. In [17] presented a comparison of SAW, TOPSIS and VIKOR. They observed that TOPSIS and SAW had identical rankings, while VIKOR produced different rankings. They concluded that both TOPSIS and VIKOR are suitable for weighing analogous problems and provide results close to reality.

The selection of best MADM method for a specific problem is a difficult task. There are many factors that should be considered before selecting a MADM method or a combination of MADM methods. Their big defect is that in a single problem, they present different results. To deal with this, some methods have been suggested [13] known as aggregation methods, where a problem with several MADM methods is ranked, and then, the final selection may be made on the basis of an aggregation of those methods results. Based on MADM literature, the reason that researchers explore new aggregation methods for decision making is to augment selection confidence of existing ranking methods. in addition to known MADM methods, aggregation methods perform the ranking of alternatives efficiently and accurately. 


\section{MADM tools}

Handover decision making can be treated as a problem where there are $n$ candidate networks, and $m$ criteria. Rows and columns of the decision matrix present the alternatives $A_{1} \ldots A_{n}$ and criteria $C_{1} \ldots C_{m}$, respectively. $a_{i j}$ defines the quantity of alternative $A_{i}$ against criterion $C_{j}$. Weights $w_{1} \quad \ldots w_{m}$ have to be positive and designated to all criteria. They define the criterion importance to the decision making.

\subsection{TOPSIS}

TOPSIS as one of the widely adapted classical MADM tools used for ranking problems was developed in order to reach non inferior solutions. It has been satisfactorily implemented in different application fields [24]. It is based on the following idea: the best alternative is supposed to have the shortest distance from the positive ideal solution (made up of the best value of each criterion disregarded the alternatives), and the longest distance from negative ideal solution (made up of the worst value of each criterion regardless of alternatives). TOPSIS is a reliable method for risk avoidance because the designers may desire a decision that not only maximises profit but also avoids risk. TOPSIS includes many steps:

step 1: decision matrix normalization

$$
p_{i j}=\left(\frac{a_{i j}}{\sqrt{\sum_{i=1}^{n} a_{i j}^{2}}}\right)
$$

step 2: weights are multiplied to the normalized matrix as follows

$$
v_{i j}=w_{j} p_{i j}
$$

step 3: positive ideal solution is $A^{+}=\left(v_{1}^{+}, \ldots, v_{j}^{+}, \ldots, v_{m}^{+}\right)$, where $v_{j}^{+}$is the best value of the $j^{\text {th }}$ attribute over all the available alternatives. Negative ideal solution is $A^{-}=\left(v_{1}^{-}, \ldots, v_{j}^{-}, \ldots, v_{m}^{-}\right)$, where $v_{j}^{-}$is the worst value of the $j^{\text {th }}$ attribute over all the available alternatives. They are computed as follows:

$$
\begin{aligned}
& A^{+}=\left\{\left(\max _{i} v_{i j} \mid j \in J\right),\left(\min _{i} v_{i j} \mid j \in J\right) \mid i=1,2, \ldots, n\right\} \\
& A^{-}=\left\{\left(\min _{i} v_{i j} \mid j \in J\right),\left(\max _{i} v_{i j} \mid j \in J\right) \mid i=1,2, \ldots, n\right\}
\end{aligned}
$$

$J\{1,2, \ldots, m\}$ and $J\{1,2, \ldots, m\}$ are the sets of criteria which need to be maximized and minimized, respectively.

step 4: the normalized euclidean distance between alternatives and ideal solutions is applied

$$
d_{i}^{+}=\sqrt{\sum_{j=1}^{m}\left(v_{i j}-v_{j}^{+}\right)^{2}} \text { and } d_{i}^{-}=\sqrt{\sum_{j=1}^{m}\left(v_{i j}-v_{j}^{-}\right)^{2}}
$$

step 5: the relative closeness $C_{i}$ to the ideal solution is computed

$$
C_{i}=\frac{d_{i}^{-}}{d_{i}^{-}+d_{i}^{+}}
$$

The best ranked alternative is the one with maximum value of $C_{i}$.

\subsection{VIKOR}

VIKOR was set up for the multi criteria optimisation of complex systems. It is a helpful tool, especially when the preference is unknown for a decision maker at the beginning of the system design. Each alternative is measured based on an aggregate function. So, the compromise ranking of alternatives is achieved by comparing the measure of closeness to the ideal solution. Any exclusion or inclusion of an alternative can influence VIKOR ranking results. This algorithm prepares a minimum of individual regret and maximum group utility for opponent and majority, respectively [20]. $v$ is the strategy weight assigned to the majority of attributes. One of VIKOR characteristics is that aggregate function always is closest to the best solutions. For TOPSIS aggregate function is not always very close to the ideal solutions. This makes VIKOR suitable for obtaining maximum profit. The VIKOR procedure is described below:

step 1: determination of aspired $\left(f_{j}^{+}\right)$and tolerable $\left(f_{j}^{-}\right)$ levels of benefit and cost criteria, respectively where $j=1,2, \ldots, m$

$$
\begin{aligned}
& f_{j}^{+}=\max _{i} a_{i j}, \quad f_{j}^{-}=\min _{i} a_{i j} \\
& f_{j}^{+}=\min _{i} a_{i j}, \quad f_{j}^{-}=\max _{i} a_{i j}
\end{aligned}
$$

step 2: calculation of utility $S_{i}$ and regret $R_{i}$ using the following where $j=1,2, \ldots m$

$$
S_{i}=\sum_{j=1}^{m} w_{j} \frac{f_{j}^{+}-f_{i j}}{f_{j}^{+}-f_{j}^{-}} \quad R_{i}=\max _{j}\left(w_{j} \frac{f_{j}^{+}-f_{i j}}{f_{j}^{+}-f_{j}^{-}}\right)
$$

step 3: The index $Q_{i}$ is calculated. $S_{\text {min }}$ and $R_{\text {min }}$ are the minimum values of $S_{i}$ and $R_{i}$, respectively. $S_{\text {max }}$ and $R_{\text {max }}$ are their maximum values, respectively.

$$
Q_{i}=v \frac{S_{i}-S_{\text {min }}}{S_{\text {max }}-S_{\text {min }}}+(1-v) \frac{R_{i}-R_{\text {max }}}{R_{\text {min }}-R_{\max }}
$$

$Q_{i}, S_{i}$, and $R_{i}$, are three ranking lists. The alternatives are arranged in a descending order in accordance with $Q_{i}$ values. They are also arranged in accordance with $S_{i}$ and $R_{i}$ values separately. The best ranked alternative $A_{1}$ is the one with minimum value of $Q_{i} . A_{1}$ is the compromise solution if:

Condition 1: $Q\left(A_{2}\right)-Q\left(A_{1}\right) \geq(1 /(n-1))$, where $A_{2}$ is the second best alternative ranked by $Q_{i}$.

Condition 2: $A_{1}$ must be also best ranked alternative by 
$S$ and/or $R$.

If one of the conditions is not fulfilled, a group of compromise solutions is proposed: $A_{1}$ and $A_{2}$ if only condition 2 is not satisfied. $A_{1}, A_{2}, \ldots, A_{m}$ if condition 1 is not satisfied. $A_{m}$ is defined by the relation $Q\left(A_{m}\right)-$ $Q\left(A_{1}\right) \leq(1 /(n-1))$.

\section{Rank Average}

Evidently, distinct decision making methods provide different results according to their hypotheses and approaches. Since seamless VH decision making is very important, it is better to use more than one method. In order to overcome this problem, we introduce an aggregate method called Rank Average. As it implicates other methods (TOPSIS and VIKOR) results and particulars, this method is able to be perfect for target access network selection. Rank Average ranks alternatives based on the average of calculated rankings of the implied MADM methods. The ranking $R_{\text {mixed }}(i)$ of the $i^{t h}$ candidate network is acquired as follow, where $k$ is the number of implied methods:

$$
R_{\text {mixed }}(i)=\frac{\sum_{k} R_{k}(i)}{k}
$$

This average ranking leads to satisfactory performance. It is accepted as invaluable because it is able to add the respective powers of every approach. In our scenario, TOPSIS and VIKOR calculate the ranking of alternatives. Then Rank Average calculates the final results for all alternatives. We choose TOPSIS and VIKOR for three reasons: (1) Each of them is advantageous and efficient for handover decision making. (2) They employ different aggregation and normalization functions. So, they give distant results for the same decision problem. For example, a selected alternative as the best by TOPSIS may be considered as the worse by VIKOR. (3) Rank Average can take advantage from their complementary powers regardless of their differences, and make efficient handover decisions.

Entropy [18][19] has been used to calculate the adequate weight of every criterion. Compared to other objective weighting methods such as bi-criteria programming, entropy has the advantages of computational simplicity and efficiency. Decision makers are also more likely to capture the analysis results more easily. Greater entropy value engender smaller weight and less importance of the criterion in the decision making process. When differences of alternatives in this particular criterion are small, there is less information provided. In other words, a criterion has less importance if all candidate materials have similar performance ratings for that attribute. This makes the results more correct and logical. Entropy determines the weights through the following steps: step 1: normalization of the decision matrix using equation (1), in order to eliminate the criteria units.

step 2: calculation of the entropy value for each criterion, where $k$ is the Boltzmann's constant

$$
E_{j}=-k \sum_{i=1}^{n} p_{i j} \ln p_{i j} \quad \text { where } k=\frac{1}{\ln n}
$$

step 3: extraction of objective criteria weights

$$
w_{j}=\frac{1-E_{j}}{\sum_{j=1}^{m}\left(1-E_{j}\right)}
$$

\section{Performance Evaluation and Results}

In this section, we evaluate Rank Average performance. To this end, we added MIH module to NS3 under which we have run simulations. We have considered a HetNet of WiFi, LTE, and WiMAX access networks. MTs are outfitted with three network devices of every access technology, and an MIH interface. MIH is needed to construct a list of local interfaces, obtain states and control their behaviour. Once the MT is switched on, the $\mathrm{VH}$ procedure is activated. In the simulation scenario, we monitor four MTs:

- MT1 runs a VoIP application while moving with a constant speed equal to $1 \mathrm{~m} / \mathrm{s}$. The VoIP application uses a G.729 codec, with $8,5 \mathrm{Kbps}$ data rate and $60 \mathrm{~B}$ packet size.

- MT2 runs a video streaming application while moving with a constant speed equal to $1 \mathrm{~m} / \mathrm{s}$. The video streaming application sends MPEG4 stream using H. 263 codec, with $16 \mathrm{Kbps}$ bit rate.

- MT3 runs web browsing application. For this, we used an HTTP traffic generator library. The generated traffic represents real world http traffic. This MT is moving across networks with a speed of $1.3 \mathrm{~m} / \mathrm{s}$.

- MT4 downloads e-mails as a background application with $8.5 \mathrm{Kbps}$ data rate and $500 \mathrm{~B}$ as packet size.

Simulation results for Rank Average, TOPSIS, and VIKOR with MIH across WiFi, WiMAX and LTE are presented in this section. The objective is to evaluate and compare these methods through some critical performance metrics: throughput, end to end delay, packet loss rate, and handover decision delay [21]. The measurements are taken every 10s. Table.1 shows the simulation parameters.

\subsection{Throughput}

Throughput figures among important QoS statistics. In our context, it is the number of bits received successfully by the MT divided by the difference between last packet reception time and the first packet transmission time. The results in figure 1 shows that Rank average is able to improve transmission throughput for real time services like VoIP and non-real 


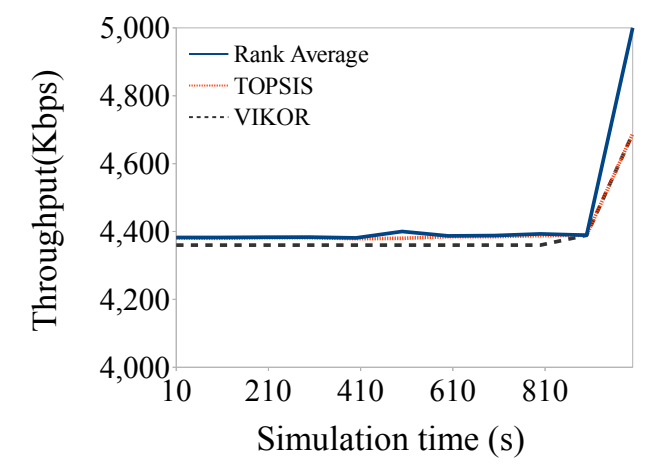

(a) VoIP

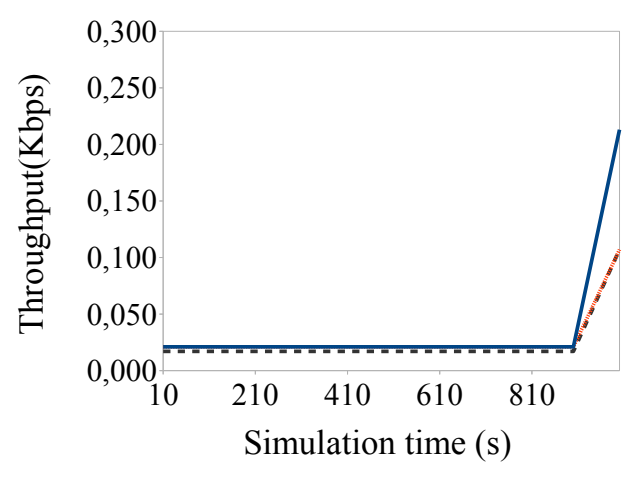

(c) Web browsing

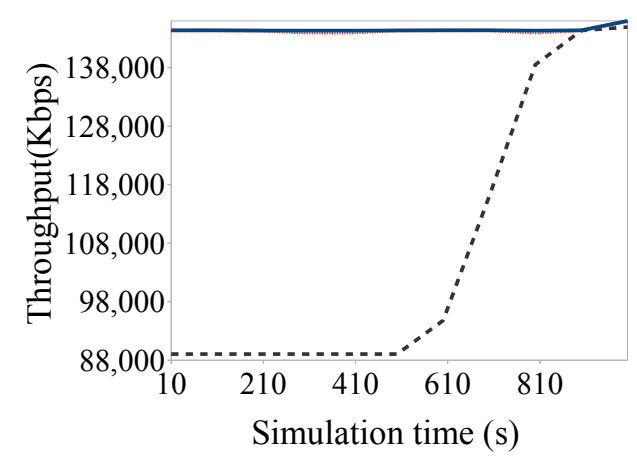

(b) Video streaming

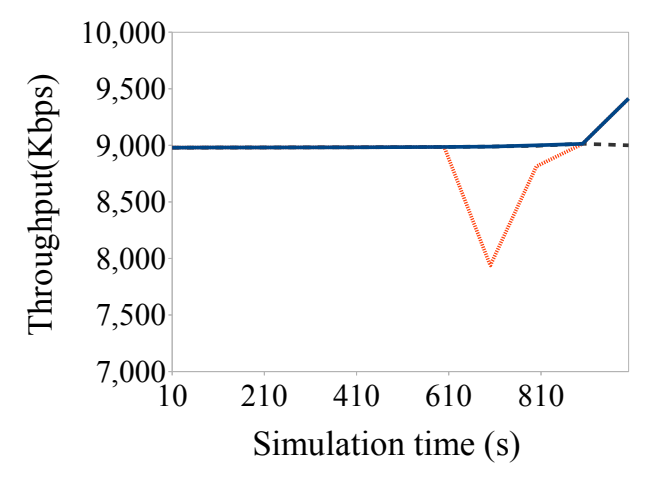

(d) E-mails download

Figure 1. MTs throughput

Table 1. Simulationparameters

\begin{tabular}{c|c}
\hline Simulationparameters & Values \\
\hline IEEE802.11 frequencybandwidth & $5 \mathrm{GHz}$ \\
IEEE802.11 transmision radius & $100 \mathrm{~m}$ \\
IEEE802.11 data rate & $20 \mathrm{Mbps}$ \\
IEEE802.16 frequencybandwidth & $5 \mathrm{GHz}$ \\
IEEE802.16 transmision radius & $600 \mathrm{~m}$ \\
IEEE802.16 channelbandwidth & $10 \mathrm{MHz}$ \\
Propagationmodel & COST231_PROPAGAION \\
IEEE802.16 modulationand coding & OFDMQAM16_12 \\
MAC/IEEE802.16CDinterval & $10 \mathrm{~s}$ \\
MAC/IEEE802.16CDinterval & $10 \mathrm{~s}$ \\
LTE uplinkbandwidth & 25 resourceblocks \\
LTE downlinlbandwidth & 25 resourceblocks \\
LTE link data rate & $10 \mathrm{Gbps}$ \\
LTE channelbandwidth & $5 \mathrm{MHz}$ \\
Maximuntransmision Power & $30.0 \mathrm{dBm}$ \\
LTE path loss model & Friis propagation \\
LTE transmision radius & $2000 \mathrm{~m}$ \\
Mobilitymodel & constantposition \\
\hline
\end{tabular}

time like downloading e-mails. Throughput offered by
Rank Average is a bit higher than that of TOPSIS and VIKOR.

\subsection{End to end delay}

End to end delay is calculated for every received packet. Figure 2 shows that Rank Average has a better end to end delay performance than TOPSIS and VIKOR. Since real time flows are very sensitive to delay, we can say that decreased delay is a potential benefit of Rank Average. Hence, it guarantees a better QoS in terms of end to end delay.

\subsection{Packet loss rate}

In order to achieve seamless $\mathrm{VH}$, it is necessary to guarantee service continuity and QoS, which means low latency and packet loss during handover. Figure 3 shows that the three evaluated approaches assure low packet loss rate. Furthermore, Rank Average guarantees null packet loss. This improves the QoS for real time services. 


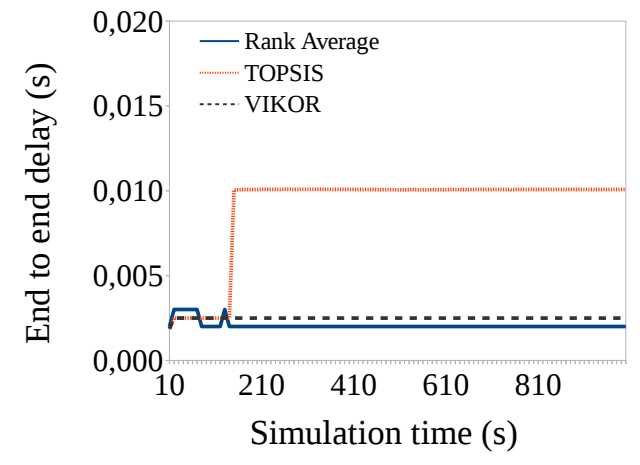

(a) VoIP

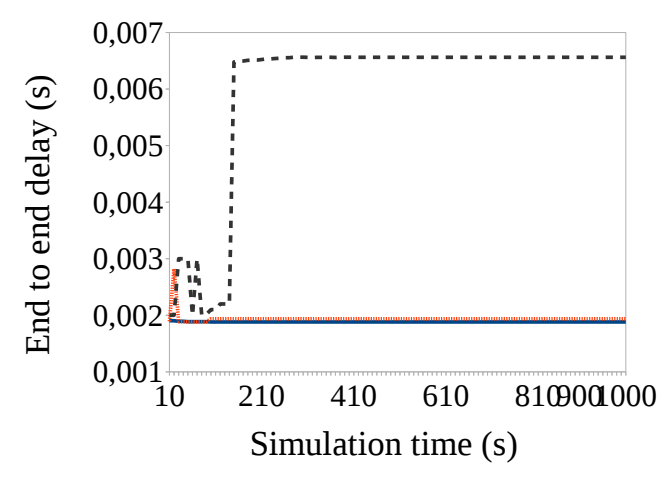

(c) Web browsing

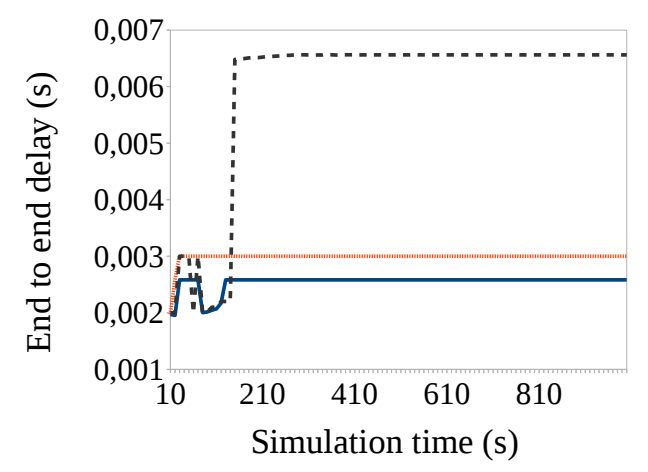

(b) Video streaming

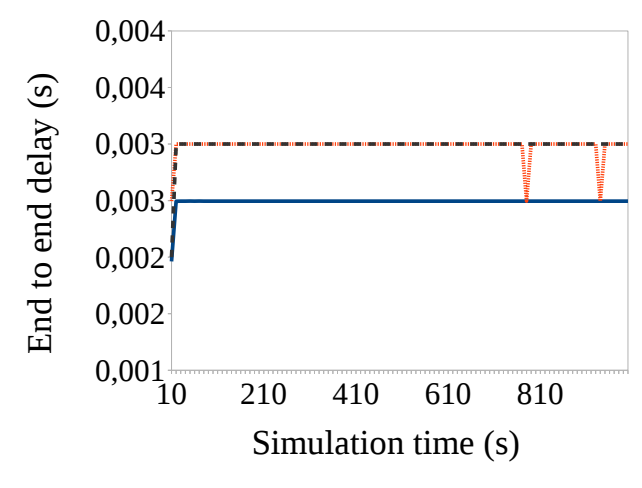

(d) E-mails download

Figure 2. Packet end to end delay between MT and its correspondentode

\subsection{Handoverdelay}

Handover delay is the time taken by the MT to make a decision and select the best access network to move to. Every time we apply Rank Average, TOPSIS, or VIKOR, we monitor the MT for 1000 s to get the number of handover occurrences, and measure decision delay. Figure 4 shows the obtained results. Number of handover events executed by VIKOR is higher compared to TOPSIS and Rank Average. For VoIP at $10 \mathrm{~s}$, web browsing at 20s, and e-mails download at 10 s, the three evaluated methods execute a handover, but Rank Average has a greater delay than TOPSIS and VIKOR. This is due to the fact that Rank Average waits for the ranking results of TOPSIS and VIKOR, then calculates the average ranking for each available access network. Although it requires more delay to decide a handover, Rank Average can achieve better performance than conventional TOPSIS and VIKOR, with respect to end to end delay, packet loss, and throughput.

Ping-pong effect is defined as the unnecessary handover to the neighbouring Base Station (BS) or Access Point (AP) that returns to original $\mathrm{BS}$ or $\mathrm{AP}$ after a very short interval of time. This unnecessary back and forth handover leads to heavy processing and loads. Rank Average compared to VIKOR reduces the number of unnecessary handovers. Thus, resources are saved, dropped calls and ping-pong effect are reduced.

\section{Conclusion}

In this paper, we employed Rank Average as an aggregation of TOPSIS and VIKOR. Rank Average method has the best performance according to simulation results, except for decision delay. It can reduce the number of unnecessary handovers, pingpong effects, end to end delay and packet loss rate, and improve throughput. That confirms the ability of Rank Average to add the powers of applied methods, and find a compromise between their proposed solutions despite their differences.

These results lead us to the necessity of reducing handover delays. Looking ahead, greater integration of artificial neural networks and fuzzy logic into a handover management scheme seem to be more promising reduced decision delays. 


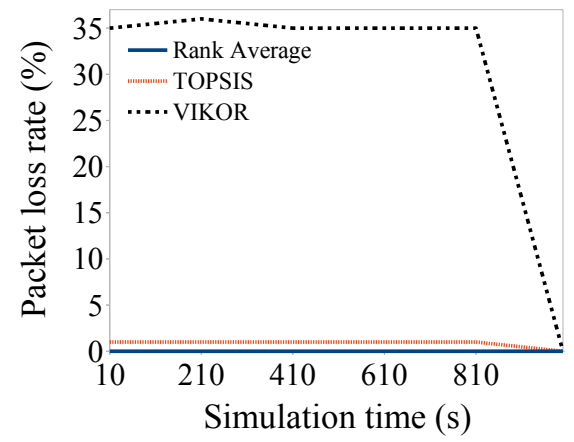

(a) VoIP

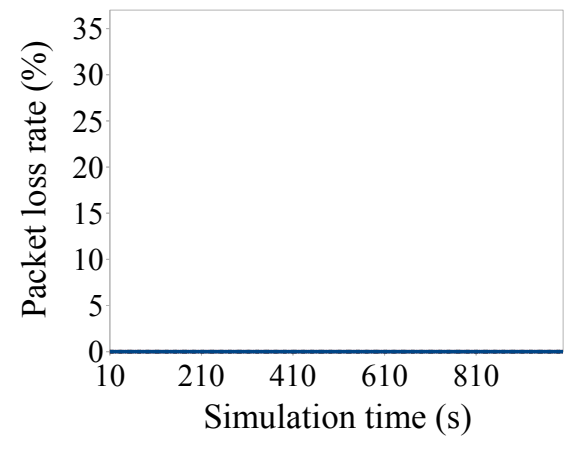

(c) Web browsing

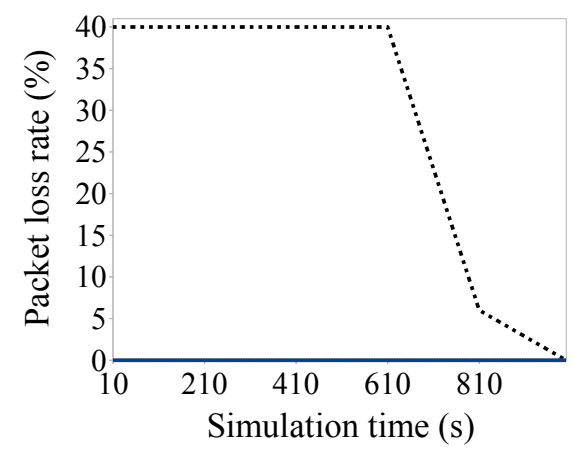

(b) Video streaming

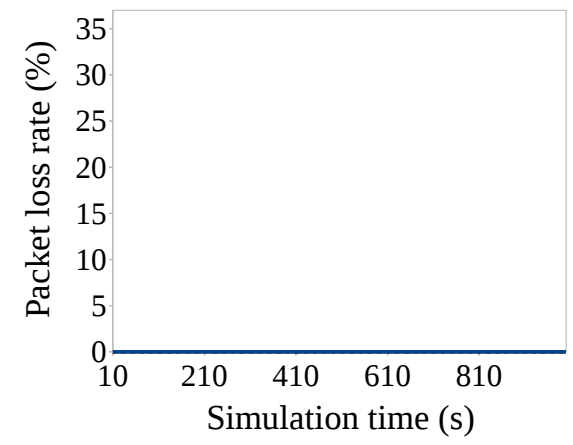

(d) E-mails download

Figure 3. Packet loss rate

\section{References}

[1] Sagar EL, Bhadla M. A survey of handover mechanism with mobility management in femtocell \& macrocell for lte. cell 2015;4(11).

[2] Ali T, Saquib M. Analysis of an instantaneous packet loss based $\mathrm{VH}$ algorithm for heterogeneous wireless networks. IEEE Transactions on Mobile Computing 2014;13(5):9921006.

[3] Sasirekha V, Chandrasekar C, Ilangkumaran M. Heterogeneous wireless network vertical handoff decision using hybrid multi-criteria decision-making technique. International Journal of Computational Science and Engineering 2015;10(3):263-80.

[4] Lahby M, Cherkaoui L, Adib A. An enhanced-topsis based network selection technique for next generation wireless networks. In: Telecommunications (ICT), 2013 20th International Conference on. IEEE; 2013, p. 1-5.

[5] Bisio I, Delucchi S, Lavagetto F, Marchese M. Performance comparison of network selection algorithms in the framework of the 802.21 standard. Journal of Networks 2015;10(01):51-9.

[6] Gul M, Celik E, Aydin N, Gumus AT, Guneri AF. A state of the art literature review of vikor and its fuzzy extensions on applications. Applied Soft Computing 2016;46:60-89.
[7] Baghla S, Bansal S. Effect of normalization techniques in vikor method for network selection in HetNets. In: Computational Intelligence and Computing Research (ICCIC), 2014 IEEE International Conference on. IEEE; 2014, p.1-6.

[8] Bhute HA, Karde P, Thakare V. AVH decision approaches in next generation wireless networks: A survey. International Journal of Mobile Network Communications \& Telematics (IJMNCT) 2014;4.

[9] Bhute HA, Karde P, Thakare V. Vertical handover decision strategies in heterogeneous wireless networks. In: nt. Conf. on Recent Trends in Information, Telecommunication and Computing, ITC. Citeseer; 2014,.

[10] Madaan J, Kashyap I. An overview of vertical handoff decision algorithm. International Journal of Computer Applications 2015;111(3).

[11] Geetika KB. Handover management in HetNets. International Journal of Scientific \& Engineering Research 2013;4(4).

[12] Munoz P, Laselva D, Barco R, Mogensen P. Dynamic traffic steering based on fuzzy q-learning approach in a multi-rat multi-layer wireless network. Computer Networks 2014;71:100-16.

[13] Rao RV. Multiple attribute decision making in the manufacturing environment. In: Decision Making in 


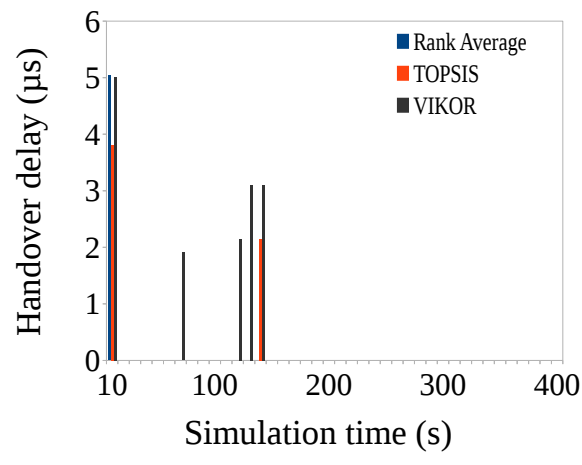

(a) VoIP

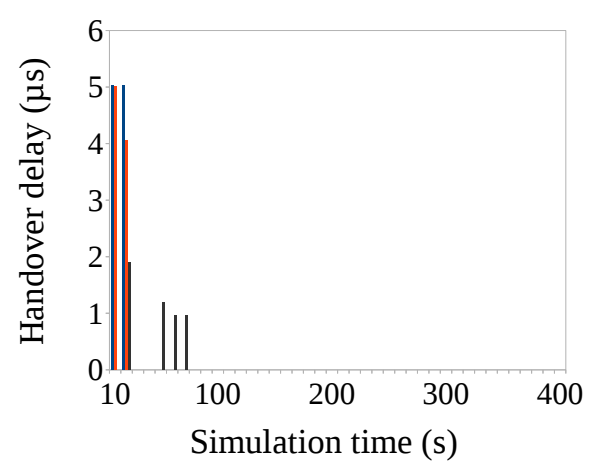

(c) Web browsing

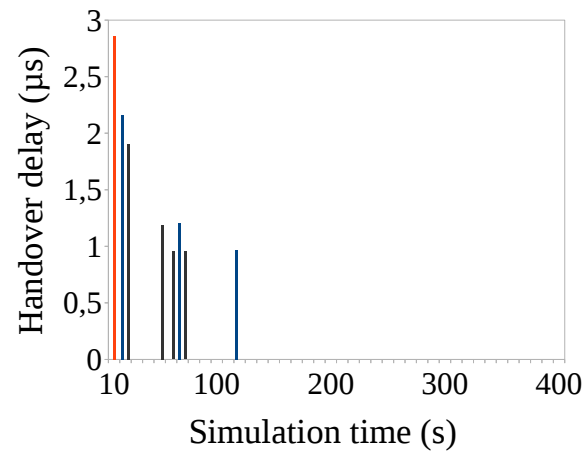

(b) Video streaming

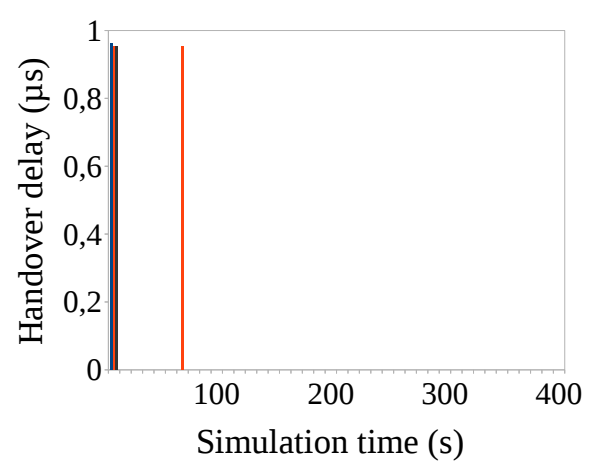

(d) E-mails download

Figure 4. VHdecisiondelay

Manufacturing Environment Using Graph Theory and Fuzzy Multiple Attribute Decision Making Methods. Springer; 2013, p.1-5.

[14] Preethi G, Chandrasekar C. A network selection algorithm based on ahp-ow a methods. In: Wireless and Mobile Networking Conference (WMNC), 2013 6th Joint IFIP. IEEE; 2013, p.1-4.

[15] Agrawal A, Jeyakumar A, Pareek N. Comparison between vertical handoff algorithms for heterogeneous wireless networks. In: Communication and Signal Processing (ICCSP), 2016 International Conference on. IEEE; 2016, p.1370-3.

[16] Zhang N, Wei G. Extension of vikor method for decision making problem based on hesitant fuzzy set. Applied Mathematical Modelling 2013;37(7):4938-47.

[17] Aguilar-Gonzalez R, Cardenas-Juarez M, Pineda-Rico U, Arce A, Latva-aho M, Stevens-Navarro E. Reducing spectrum handoffs and energy switching consumption of madm-based decisions in cognitive radio networks. Mobile Information Systems 2016;2016.

[18] Jiang W, Shen P, Liu F, Fang X. An interactive group decision making approach based on satisfaction degree. In: LISS 2014. Springer; 2015, p.1249-54.

[19] Zamri N, Abdullah L. A new linguistic variable in interval type-2 fuzzy entropy weight of a decision making method. Procedia Computer Science 2013;24:42-53.

[20] Dou Y, Zhang P, Jiang J, Yang K, Chen Y. Mcdm based on reciprocal judgment matrix: a comparative study of e-vikor and e-topsis algorithmic methods with interval numbers. Appl Math 2014;8(3):1401-11.

[21] Wu J, Cheng B, Yuen C, Shang Y, Chen J. Distortionaware concurrent multipath transfer for mobile video streaming in heterogeneous wireless networks. IEEE Transactions on Mobile Computing 2015;14(4):688-701.

[22] Budiyanto S, Asvial M, Gunawan D. Performance Analysis of Genetic Zone Routing Protocol Combined With Vertical Handover Algorithm for 3G-WiFi Offload. Journal of ICT Research and Applications. 2014 May $1 ; 8(1): 49-63$.

[23] Chai J, Liu JN, Ngai EW. Application of decision-making techniques in supplier selection: A systematic review of literature. Expert Systems with Applications. 2013 Aug 31;40(10):3872-85.

[24] Jingmeng B, Zeng B, Zhang J, Dunnan L, Ming Z. Benefits comprehensive evaluation of demand response in SDN based on distant Grey Relational Analysis-Technique for Order Preference by Similarity to Ideal Solution. InPower and Energy Engineering Conference (APPEEC), 2016 IEEE PES Asia-Pacific 2016 Oct 25 (pp.843-851). IEEE. 